\title{
Morphisms generating antipalindromic words
}

\author{
Petr Ambrož, Zuzana Masáková, Edita Pelantová \\ Faculty of Nuclear Sciences and Physical Engineering \\ Czech Technical University in Prague \\ Trojanova 13, 12000 Praha 2, Czech Republic
}

\begin{abstract}
We introduce two classes of morphisms over the alphabet $A=\{0,1\}$ whose fixed points contain infinitely many antipalindromic factors. An antipalindrome is a finite word invariant under the action of the antimorphism $\mathrm{E}:\{0,1\}^{*} \rightarrow\{0,1\}^{*}$, defined by $\mathrm{E}\left(w_{1} \cdots w_{n}\right)=\left(1-w_{n}\right) \cdots\left(1-w_{1}\right)$. We conjecture that these two classes contain all morphisms (up to conjugation) which generate infinite words with infinitely many antipalindromes. This is an analogue to the famous HKS conjecture concerning infinite words containing infinitely many palindromes. We prove our conjecture for two special classes of morphisms, namely (i) uniform morphisms and (ii) morphisms with fixed points containing also infinitely many palindromes.
\end{abstract}

Keywords: palindromes; antipalindromes; uniform morphisms; class $\mathcal{P}$.

\section{Introduction}

Palindromic words are infinite words over a finite alphabet $A$ which contain arbitrarily long palindromes. Recall that a palindrome is a finite word $w$ which is read the same backwards and forwards, i.e., $w=w_{1} w_{2} \cdots w_{n}=w_{n} w_{n-1} \cdots w_{1}$. Palindromic words have been extensively studied since the observation of Hof, Knill and Simon [10] that they can be used for construction of aperiodic potentials of discrete Schrödinger operators with purely singular continuous spectrum. Such Schrödinger operators seem to describe well the behaviour of one-dimensional structures known under the name quasicrystals.

A large class of palindromic words is the family of Sturmian words defined as infinite aperiodic words with minimal complexity. One of Sturmian words is the Fibonacci word $\boldsymbol{f}=010010100100101001 \cdots$. The word $\boldsymbol{f}$ can be constructed by iterating the rewriting rule $0 \mapsto 01,1 \mapsto 0$, i.e.,

$$
0 \mapsto 01 \mapsto 010 \mapsto 01001 \mapsto 01001010 \mapsto \cdots
$$

Note that the word in $i$-th iteration is a prefix of the word in iteration $i+1$ and the infinite word $\boldsymbol{f}$ is defined naturally. The construction can be formalized using the notion of a homomorphism over the free monoid $A^{*}$ of all words over a finite alphabet (equipped with the operation of concatenation and the empty word as the neutral element). In the context of combinatorics on words, the homomorphisms are called just morphisms. The Fibonacci word $\boldsymbol{f}$ is thus an example of an infinite word fixed by the morphism $\varphi:\{0,1\}^{*} \rightarrow\{0,1\}^{*}$, defined by $\varphi(0)=01, \varphi(1)=0$. 
The authors of [10] conjecture that any palindromic fixed point of a morphism can be generated by a morphism conjugated to an element of the so-called class $\mathcal{P}$ - a family of morphisms in a special form, namely that $\varphi(a)=p_{a} q$ where $q$ and $p_{a}$ for $a \in A$ are palindromes. This - the so-called HKS conjecture - has been proven for binary words by Tan in [25]. Partial results for infinite words over larger alphabets have been also given. For example, the HKS conjecture is proved in [1] for periodic words, in [14] for fixed points of marked morphisms, and in [16] for words coding non-degenerated exchange of three intervals. In [9], a modified version of HKS conjecture has been proven for rich words.

The definition of a palindrome can be formulated using the notion of an antimorphism over the monoid $A^{*}$. A mapping $\eta: A^{*} \rightarrow A^{*}$ is an antimorphism if $\eta(v w)=\eta(w) \eta(v)$ for any pair of words $v, w \in A^{*}$. A palindrome is a finite word invariant under the mirror image antimorphism $\mathrm{R}$. Words invariant under other involutive antimorphisms are called generalized palindromes or pseudopalindromes. In the particular case of a binary alphabet, the only involutive antimorphism other than $\mathrm{R}$ is the exchange map $\mathrm{E}$. The words $w$ such that $\mathrm{E}(w)=w$ are called antipalindromes. For example, the shortest nonempty antipalindrome is 01 , as $E(01)=E(1) E(0)=01$. An infinite word containing infinitely many such antipalindromes is called antipalindromic. The well known ThueMorse word $\boldsymbol{t}=0110100110010110 \cdots$, both fixed points of the morphism $\Theta: 0 \mapsto$ $01,1 \mapsto 10$, can serve as an example. A large class of antipalindromic words is given by complementary symmetric Rote words, see [2]. These words are, however, not fixed by any non-identical morphism, see [17.

Our aim is to study a modification of the HKS conjecture to the case of antipalindromes. We define two classes of morphisms $\mathcal{A}_{1}, \mathcal{A}_{2}$ such that any fixed point of a morphism in any of these classes is antipalindromic. We conjecture that classes $\mathcal{A}_{1}, \mathcal{A}_{2}$ contain (up to conjugacy) all primitive morphisms with antipalindromic fixed points. The conjecture is supported by our results formulated as Theorem 34 and Theorem 36 which state that

- if a binary uniform morphism $\varphi$ (i.e., such that the words $\varphi(0), \varphi(1)$ are of the same length) has an antipalindromic fixed point, then $\varphi$ or $\varphi^{2}$ is (up to conjugacy) equal to a morphism in class $\mathcal{A}_{1}$;

- if a morphism $\varphi$ is primitive and its fixed point contains infinitely many of both palindromes and antipalindromes, then $\varphi$ or $\varphi^{2}$ belongs to $\mathcal{A}_{1} \cap \mathcal{P}$ or $\mathcal{A}_{2} \cap \mathcal{P}$.

The situation can be summarized in a diagram displayed in Figure 1 which shows intersection of the above mentioned classes $\mathcal{P}, \mathcal{A}_{1}, \mathcal{A}_{2}$.

This paper is organized as follows. In Section 2 we recall a number of necessary notions and tools from combinatorics on words. In particular, we recall several results on equations on words and on the structure of bispecial factors in languages of morphic words. In Section 3 we define classes $\mathcal{A}_{1}, \mathcal{A}_{2}$ and show that their fixed points are antipalindromic. We also characterize morphisms in classes $\mathcal{P} \cap \mathcal{A}_{1}$ and $\mathcal{P} \cap \mathcal{A}_{2}$. Section 4 describes eventually periodic words which contain infinitely many antipalindromes and those which contain infinitely many of both palindromes and antipalindromes. Properties of languages of infinite antipalindromic aperiodic words are studied in Section 5 . The main results (Theorems 34 and 36) are stated in Section 6. Theorem 34 for uniform mosphism is also proved there. The proof of Theorem 36 which concerns non-uniform 


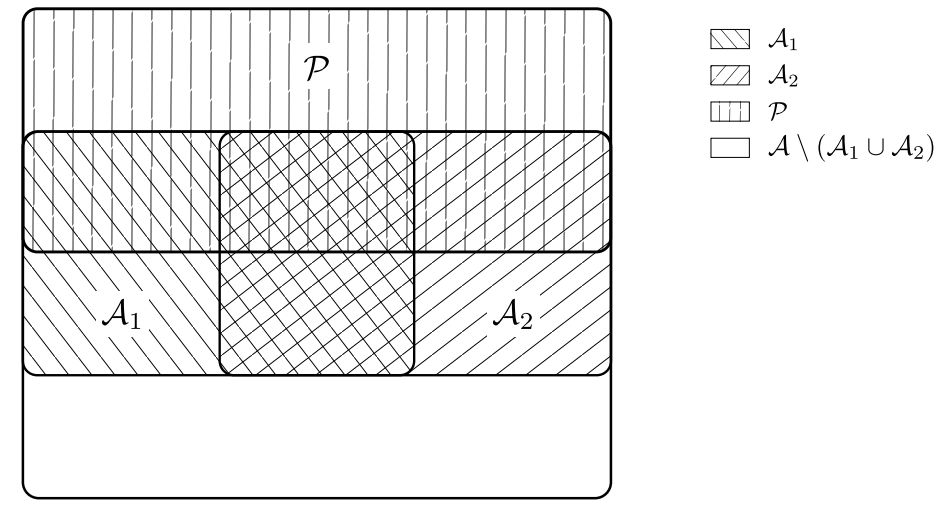

Figure 1: Relation of classes $\mathcal{A}_{1}, \mathcal{A}_{2}$ and $\mathcal{P}$. Denoting by $\mathcal{A}$ the set of all primitive morphisms with antipalindromic fixed points, we conjecture that the set $\mathcal{A} \backslash\left(\mathcal{A}_{1} \cup \mathcal{A}_{2}\right)$ is empty.

morphisms is very technical and requires a number of auxiliary results. They are collected in Section 7. Finally, we include comments and open problems.

\section{Preliminaries}

\section{$2.1 \quad$ Finite Words}

Let $A$ be a finite set called alphabet, its elements are called letters. A finite sequence $w=w_{1} \cdots w_{n}$ such that $w_{i} \in A$ for every $i=1,2, \ldots, n$ is called a word over $A$. The length (the number of elements) of $w=w_{1} \cdots w_{n}$ is denoted by $|w|=n$. The notation $|w|_{a}$ is used for the number of occurrences of the letter $a$ in $w$. The word of length zero - the so called empty word - is denoted by $\varepsilon$. The set of all finite words over $A$ equipped with the operation concatenation of words forms the free monoid $A^{*}$, its neutral element is $\varepsilon$.

Let $w=p u s$ for some $p, u, s \in A^{*}$. Then $u, p$, and $s$ are called a factor, a prefix, and a suffix of $w$, respectively. Let $w \in A^{*}$ and $r \in \mathbb{N}$ then $w^{r}$ denotes the $r$-th power of $w$, i.e., $w^{r}=\underbrace{w w \cdots w}_{r \text {-times }}$. A word $v \in A^{*}$ is called primitive if for each $w \in A^{*}$ and $r \in \mathbb{N}$ the equality $v=w^{r}$ implies that $w=v$ and $r=1$.

A mapping $\varphi: A^{*} \rightarrow A^{*}$ is called a morphism (over $A$ ) if $\varphi(u v)=\varphi(u) \varphi(v)$ for every $u, v \in A^{*}$; it is called an antimorphism if $\varphi(u v)=\varphi(v) \varphi(u)$ for every $u, v \in A^{*}$. Obviously, both morphism and antimorphism are fully defined by providing $\varphi(a)$ for all $a \in A$.

A morphism $\varphi: A^{*} \rightarrow A^{*}$ is said to be primitive if there exists $k \in \mathbb{N}$ such that for every pair $a, b \in A$ the letter $a$ occurs in the word $\varphi^{k}(b)$. A morphism $\varphi: A^{*} \rightarrow A^{*}$ is called uniform if for every pair $a, b \in A$ we have $|\varphi(a)|=|\varphi(b)|$.

Throughout the paper, we use two important antimorphisms $\mathrm{R}$ and $\mathrm{E}$. The antimorphism $\mathrm{R}: A^{*} \rightarrow A^{*}$, called mirror image map, is defined as $\mathrm{R}(a)=a$ for every $a \in A$, that is, $\mathrm{R}\left(w_{1} \cdots w_{n}\right)=w_{n} \cdots w_{1}$. The antimorphism $\mathrm{E}:\{0,1\}^{*} \rightarrow\{0,1\}^{*}$ is the exchange map defined by $\mathrm{E}(0)=1$ and $\mathrm{E}(1)=0$. Note that both antimorphisms are involutions, 
i.e., $\mathrm{R}^{2}=\mathrm{E}^{2}=\mathrm{id}$. Let $w$ be a word, if $\mathrm{R}(w)=w$ then $w$ is said to be a palindrome, if $\mathrm{E}(w)=w$ then $w$ is called antipalindrome. Note that an antipalindrome necessarily has even length. The only word which is both palindrome and antipalindrome is the empty word $\varepsilon$.

One can easily check the following properties of antimorphisms R, E and Thue-Morse morphism $\Theta$ given by $\Theta(0)=01$ and $\Theta(1)=10$.

Observation 1. Let $w \in\{0,1\}^{*}$. Then

i) $w$ is a palindrome $\Leftrightarrow \mathrm{E}(w)$ is a palindrome,

ii) $w$ is an antipalindrome $\Leftrightarrow \mathrm{E}(w)$ is an antipalindrome,

iii) $\Theta \mathrm{R}=\mathrm{E} \Theta$,

iv) $\Theta(w)$ is a palindrome $\Leftrightarrow w$ is an antipalindrome,

v) $\Theta(w)$ is an antipalindrome $\Leftrightarrow w$ is a palindrome.

In our considerations we will repeatedly use several known results on the solutions of equations on words. The statements of these results are summarized in the following proposition, the proofs can be found in [15, 4, 20].

Proposition $2([15,4,20])$. Let $x, y, z \in A^{*}$.

i) If $x y=y x$, then there exist $u \in A^{*}$ and $i, j \in \mathbb{N}$ such that $x=u^{i}$ and $y=u^{j}$.

ii) If $x y=y z$ and $x \neq \varepsilon$, then there exist $u, v \in A^{*}$ and $i \in \mathbb{N}$ such that $x=u v$, $y=(u v)^{i} u$, and $z=v u$.

iii) If $x, y \in\{0,1\}^{*}$ are nonempty palindromes and $x y$ is an antipalindrome, then there exist a palindrome $u \in\{0,1\}^{*}$ and $i, j \in \mathbb{N}$ such that $x=(u \mathrm{E}(u))^{i} u$ and $y=$ $(\mathrm{E}(u) u)^{j} \mathrm{E}(u)$.

Finally, let us recall the Fine-Wilf theorem.

Theorem 3 ([15]). Let $x, y \in A^{*}$. If $w$ is a prefix of both $x^{r}$ and $y^{r}$ for some $r \in \mathbb{N}$ and if $|w| \geq|x|+|y|-\operatorname{gcd}\{|x|,|y|\}$, then there is $z \in A^{*}$ such that $x=z^{i}$ and $y=z^{j}$ for some $i, j \in \mathbb{N}$.

\subsection{Infinite words}

An infinite word over an alphabet $A$ is an infinite sequence $\boldsymbol{u}=u_{0} u_{1} u_{2} \cdots$ of letters from $A$ (i.e., $u_{i} \in A$ for every $i \in \mathbb{N}$ ). The set of all infinite words over $A$ is denoted $A^{\mathbb{N}}$. A finite word $w \in A^{*}$ of length $|w|=n$ is called a factor of $\boldsymbol{u}$ if there is an index $i \in \mathbb{N}$ such that $w=u_{i} u_{i+1} \cdots u_{i+n-1}$. The index $i$ is called an occurrence of $w$ in $\boldsymbol{u}$. The set of all factors of $\boldsymbol{u}$ is called the language of $\boldsymbol{u}$, denoted $\mathcal{L}(\boldsymbol{u})$. We say that $\mathcal{L}(\boldsymbol{u})$ is closed under $\mathrm{R}$, if $w \in \mathcal{L}(\boldsymbol{u})$ implies $\mathrm{R}(w) \in \mathcal{L}(\boldsymbol{u})$. Analogously, $\mathcal{L}(\boldsymbol{u})$ is closed under $\mathrm{E}$ if $w \in \mathcal{L}(\boldsymbol{u})$ gives $\mathrm{E}(w) \in \mathcal{L}(\boldsymbol{u})$.

An infinite word $\boldsymbol{u}$ is called eventually periodic if there exist $v, w \in A^{*}, w \neq \varepsilon$ such that $\boldsymbol{u}=v w^{\infty}$, where $w^{\infty}$ denotes an infinite repetition of $w$. If, moreover, $v=\varepsilon$, then $\boldsymbol{u}$ is purely periodic. An infinite word which is not eventually periodic is called aperiodic. An infinite word $\boldsymbol{u}$ is called recurrent if each factor $w \in \mathcal{L}(\boldsymbol{u})$ has an infinite number of occurrences in $\boldsymbol{u}$. If there is a number $r$ for all $n \in \mathbb{N}$ such that each factor of $\boldsymbol{u}$ of length 
$n$ has at least one occurrence in the set $\{k, k+1, \ldots, k+r-n\}$ for every $k \in \mathbb{N}$, then $\boldsymbol{u}$ is called uniformly recurrent and the mapping $n \mapsto r(n)$, where $r(n)$ is the minimal $r$ with the above property, is called the recurrence function of $\boldsymbol{u}$.

The domain of a morphism $\varphi: A^{*} \rightarrow A^{*}$ can be naturally extended to infinite words by $\varphi(\boldsymbol{u})=\varphi\left(u_{0} u_{1} u_{2} \cdots\right)=\varphi\left(u_{0}\right) \varphi\left(u_{1}\right) \varphi\left(u_{2}\right) \cdots$. If $\varphi(\boldsymbol{u})=\boldsymbol{u}$, then $\boldsymbol{u}$ is called a fixed point of $\varphi$.

A morphism $\varphi$ is called a substitution if it has the following property: there are $a \in A$ and $w \in A^{*}, w \neq \varepsilon$ such that $\varphi(a)=a w$ and $\left|\varphi^{n}(a)\right|$ tends to infinity with growing $n$. Obviously, a substitution $\varphi$ has at least one fixed point, namely $\boldsymbol{u}=a w \varphi(w) \varphi^{2}(w) \cdots$.

Let $\varphi, \psi$ be morphisms over $A$. We say that $\varphi$ is left conjugate to $\psi$ (or equivalently that $\psi$ is right conjugate to $\varphi$ ) if there is $q \in A^{*}$ such that $q \varphi(w)=\psi(w) q$ for every $w \in A^{*}$. In such a case the word $q$ is called the conjugacy word. If, moreover, $\varphi$ is the only left conjugate to itself, we say that $\varphi$ is the leftmost conjugate to $\psi$, denoted by $\psi_{L}$. The rightmost conjugate to a morphism $\psi$, denoted by $\psi_{R}$, is defined analogously.

Example 4. Let $\psi:\{a, b\}^{*} \rightarrow\{a, b\}^{*}$ be defined by $\psi(a)=a b a a b$ and $\psi(b)=a b$. Then $\psi_{L}(a)=a b a b a, \psi_{L}(b)=b a, \psi_{R}(a)=a b a b a$, and $\psi_{R}(b)=a b$. Clearly $\psi_{L}$ and $\psi_{R}$ are also conjugate morphisms and their conjugacy word is equal to $q=a b a b a$.

If a morphism $\psi$ is a conjugate to itself via a nonempty conjugacy word $q$ then $\psi$ is called cyclic morphism and it has a unique fixed point, namely $q^{\infty}$. Otherwise, $\psi$ is called acyclic. Any acyclic morphism has a leftmost and a rightmost conjugate.

Let $\operatorname{fst}(w)$ and $\operatorname{lst}(w)$ denote the first and the last letter of $w$, respectively. Let $\psi$ be an acyclic morphism over a binary alphabet then obviously

$$
\begin{array}{ll}
\operatorname{fst}\left(\psi_{L}(a)\right) \neq \operatorname{fst}\left(\psi_{L}(b)\right) & \text { if } a \neq b, \\
\operatorname{lst}\left(\psi_{R}(a)\right) \neq \operatorname{lst}\left(\psi_{R}(b)\right) & \text { if } a \neq b .
\end{array}
$$

A morphism over an arbitrary alphabet $A$ satisfying (1) for all $a, b \in A, a \neq b$ is called marked. Thus a binary acyclic morphism is marked.

The following proposition summarizes several important properties of fixed points of primitive morphisms, for proofs see [7, 21].

Proposition 5. Let $\varphi: A^{*} \rightarrow A^{*}$ be a primitive morphism and let $\boldsymbol{u}$ be its fixed point. Then

i) $\boldsymbol{u}$ is uniformly recurrent;

ii) if $\psi$ is a conjugate to $\varphi$ then $\psi$ is primitive;

iii) if $\psi$ is a conjugate to $\varphi$ and $\boldsymbol{v}$ is a fixed point of $\psi$ then $\mathcal{L}(\boldsymbol{v})=\mathcal{L}(\boldsymbol{u})$;

iv) for each $a \in A$ the following limit, called uniform frequency of the letter a in $\boldsymbol{u}$, exists

$$
\rho_{a}:=\lim _{\substack{|w| \rightarrow \infty \\ w \in \mathcal{L}(\boldsymbol{u})}} \frac{|w|_{a}}{|w|} .
$$

The stabilizer [12] of an infinite word $\boldsymbol{u} \in A^{\mathbb{N}}$ is the set

$$
\operatorname{Stab}(\boldsymbol{u})=\{\varphi \text { a morphism over } A: \varphi(\boldsymbol{u})=\boldsymbol{u}\} .
$$

Clearly, $\operatorname{Stab}(\boldsymbol{u})$ is closed under the composition of morphisms and the identity morphism belongs to $\operatorname{Stab}(\boldsymbol{u})$, i.e., $\operatorname{Stab}(\boldsymbol{u})$ is a monoid. An infinite word $\boldsymbol{u}$ is called rigid if there 
exists a morphism $\varphi: A^{*} \rightarrow A^{*}$ such that $\operatorname{Stab}(\boldsymbol{u})=\left\{\varphi^{k}: k \in \mathbb{N}\right\}$. In general, it is a difficult task to find the stabilizer of an infinite word. Examples of classes of words known to be rigid are Sturmian words [23], Prouhet words [24], and fixed points of epistandard morphisms [12].

An infinite word $\boldsymbol{u}$ is called palindromic if $\mathcal{L}(\boldsymbol{u})$ contains an infinite number of palindromes. If $\boldsymbol{u}$ is a uniformly recurrent word which is palindromic, then $\mathcal{L}(\boldsymbol{u})$ is closed under R. Similarly, an infinite word $\boldsymbol{u}$ is called antipalindromic if $\mathcal{L}(\boldsymbol{u})$ contains an infinite number of antipalindromes. The language $\mathcal{L}(\boldsymbol{u})$ of a uniformly recurrent antipalindromic word $\boldsymbol{u}$ is closed under E.

Tan 25] proved that a fixed point of a primitive binary morphism $\varphi$ is palindromic if and only if $\varphi$ or $\varphi^{2}$ is conjugate to a morphism in the so-called class $\mathcal{P}$.

Definition 6. A primitive morphism $\psi: A^{*} \rightarrow A^{*}$ belongs to class $\mathcal{P}$ if there is a palindrome $p \in A^{*}$ such that for each $a \in A$

$$
\psi(a)=p q_{a}, \quad \text { where } q_{a} \in A^{*} \text { is a palindrome. }
$$

One can check whether a morphism $\psi$ belongs to class $\mathcal{P}$ by means of the morphism assigning to any letter $a$ the reversal of the word $\psi(a)$. The verification is based on the following proposition, which has been proved in [25] for binary morphisms and in [14] for morphisms over multilateral alphabets.

Proposition 7 ([25, 14]). Let $\psi$ be a binary acyclic morphism. Then $\psi$ is conjugate to a morphism in class $\mathcal{P}$ if and only if $\mathrm{R}\left(\psi_{R}(a)\right)=\psi_{L}(a)$ for $a \in\{0,1\}$.

\subsection{Special factors}

Let $\boldsymbol{u}$ be an infinite word over $A$. A factor $w \in \mathcal{L}(\boldsymbol{u})$ is called right special if there exist two different letters $a, b \in A$ such that $w a, w b \in \mathcal{L}(\boldsymbol{u})$. Analogically, $w$ is called left special if there exist two different letters $c, d \in A$ such that $c w, d w \in \mathcal{L}(\boldsymbol{u})$. A factor $w$ is called bispecial if it is both left and right special. An infinite word $\boldsymbol{u}$ is aperiodic if for every $n \in \mathbb{N}$ there is a left special factor of length $n$ and a right special factor of length $n$ in $\mathcal{L}(\boldsymbol{u})$.

Bispecial factors in fixed points of morphisms were described by Klouda [11 for a broad class of morphisms. The corollaries of said description for marked morphisms were formulated by Labbé and Pelantová [14, Proposition 28]. We give here a simplified version for binary morphisms.

Theorem 8. Let $\varphi$ be a primitive binary morphism with an aperiodic fixed point $\boldsymbol{u}$. Let $\varphi_{L}$ and $\varphi_{R}$ be the leftmost and the rightmost conjugate to $\varphi$, respectively, and let $q$ be their conjugacy word, i.e., $\varphi_{R}(a) q=q \varphi_{L}(a)$ for $a=0,1$. Then

i) For each bispecial factor $w \in \mathcal{L}(\boldsymbol{u})$ the word $\Phi(w):=\varphi_{R}(w) q$ is also a bispecial factor in $\mathcal{L}(\boldsymbol{u})$.

ii) There is a finite set of bispecial factors - called initial bispecial factors - such that every bispecial factor in $\mathcal{L}(\boldsymbol{u})$ is equal to $\Phi^{k}(w)$ for some initial bispecial $w$ and some $k \in \mathbb{N}$. 


\section{Antipalindromic morphisms}

The problem of antipalindromic fixed points of morphisms was already studied by Labbé in [13]. He defines the so-called class $\mathcal{E}-\mathcal{P}$ as the set of binary morphisms of the form

$$
\varphi(a)=p p_{a}, \quad a=0,1, \quad \text { where } p, p_{0}, p_{1} \text { are antipalindromes. }
$$

Class $\mathcal{E}$ - $\mathcal{P}$ is a direct analogy of class $\mathcal{P}$. Morphisms in $\mathcal{E}$-P $\mathcal{P}$ do not necessarily generate antipalindromic fixed points (as can be seen in Example 35), nevertheless, class $\mathcal{E}$ - $\mathcal{P}$ is useful in the formulation of the problem.

Here we define two classes of morphisms $\mathcal{A}_{1}, \mathcal{A}_{2}$ and show that they both generate antipalindromic fixed points. We explain their relation to class $\mathcal{E}-\mathcal{P}$.

\subsection{Class $\mathcal{A}_{1}$ - uniform morphisms}

The first class contains uniform morphisms.

Definition 9. A morphism $\varphi:\{0,1\}^{*} \rightarrow\{0,1\}^{*}$ belongs to class $\mathcal{A}_{1}$ if there exist words $\mathfrak{p}, \mathfrak{s} \in\{0,1\}^{*}$ such that $\mathfrak{p} \neq \varepsilon, \mathfrak{s}$ is an antipalindrome, and

$$
\varphi(0)=\mathfrak{p} \mathfrak{s}, \quad \varphi(1)=\mathrm{E}(\mathfrak{p}) \mathfrak{s} .
$$

Note that morphisms in class $\mathcal{A}_{1}$ are primitive, except the case $\varphi(0)=0^{k}, \varphi(1)=1^{k}$, which produces trivial fixed points $0^{\infty}, 1^{\infty}$. A primitive morphism $\varphi$ in class $\mathcal{A}_{1}$ satisfies $|\varphi(w)|>|w|$ for every finite word $w \in\{0,1\}^{*}$.

Remark 10. Stated in our notation, Labbé [13, Lemme 3.21] shows that a uniform morphism $\varphi$ is in class $\mathcal{A}_{1}$ if and only if $\varphi \Theta$ belongs to class $\mathcal{E}-\mathcal{P}$.

Labbé also shows that morphisms in class $\mathcal{A}_{1}$ have antipalindromic fixed points. We include this result and its demonstration for self-consistence.

Proposition 11. Let $\varphi$ be a primitive morphism in class $\mathcal{A}_{1}$ and let $\boldsymbol{u}$ be its fixed point. Then $\mathcal{L}(\boldsymbol{u})$ contains infinitely many antipalindromes.

First we state a simple lemma.

Lemma 12. Let $\varphi$ be a morphism in class $\mathcal{A}_{1}$ and let $w \in\{0,1\}^{*}$. Then

$$
\mathrm{E}(\mathfrak{s} \varphi(w))=\mathfrak{s} \varphi(\mathrm{E}(w))
$$

where $\mathfrak{s}$ is the suffix of $\varphi(0)$ from Definition 9 .

Proof. We proceed by induction on the length of $w$. Suppose first that $|w|=1$. If $w=0$ then

$$
\mathrm{E}(\mathfrak{s} \varphi(0))=\mathrm{E}(\varphi(0)) \mathrm{E}(\mathfrak{s})=\mathrm{E}(\mathfrak{p s}) \mathfrak{s}=\mathfrak{s} \mathrm{E}(\mathfrak{p}) \mathfrak{s}=\mathfrak{s} \varphi(1)=\mathfrak{s} \varphi(\mathrm{E}(0)) .
$$

Otherwise $w=1$ and then

$$
\mathrm{E}(\mathfrak{s} \varphi(1))=\mathrm{E}(\varphi(1)) \mathrm{E}(\mathfrak{s})=\mathrm{E}(\mathrm{E}(\mathfrak{p}) \mathfrak{s}) \mathfrak{s}=\mathfrak{s p \mathfrak { s }}=\mathfrak{s} \varphi(0)=\mathfrak{s} \varphi(\mathrm{E}(1)) .
$$


Now let $|w|>1$, i.e., $w=w_{1} \cdots w_{n}$. We have

$$
\begin{aligned}
\mathrm{E}\left(\mathfrak{s} \varphi\left(w_{1} w_{2} \cdots w_{n}\right)\right) & =\mathrm{E}\left(\varphi\left(w_{2} \cdots w_{n}\right)\right) \mathrm{E}\left(\mathfrak{s} \varphi\left(w_{1}\right)\right)= \\
& \stackrel{(1)}{=} \mathrm{E}\left(\varphi\left(w_{2} \cdots w_{n}\right)\right) \mathfrak{s} \varphi\left(\mathrm{E}\left(w_{1}\right)\right)= \\
& \stackrel{(2)}{=} \mathrm{E}\left(\mathfrak{s} \varphi\left(w_{2} \cdots w_{n}\right)\right) \varphi\left(\mathrm{E}\left(w_{1}\right)\right)= \\
& \stackrel{(3)}{=} \mathfrak{s} \varphi\left(\mathrm{E}\left(w_{2} \cdots w_{n}\right)\right) \varphi\left(\mathrm{E}\left(w_{1}\right)\right)=\mathfrak{s} \varphi\left(\mathrm{E}\left(w_{1} \cdots w_{n}\right)\right),
\end{aligned}
$$

where we have used (1) validity of the statement for $|w|=1,(2)$ the fact that $\mathfrak{s}$ is an antipalindrome, (3) induction hypothesis for $|w|=n-1$.

Proof of Proposition 11. Let $w \in \mathcal{L}(\boldsymbol{u})$ be an antipalindrome. Lemma 12 implies that the word $\mathfrak{s} \varphi(w)$ is also an antipalindrome. Indeed, $\mathrm{E}(\mathfrak{s} \varphi(w))=\mathfrak{s} \varphi(\mathrm{E}(w))=\mathfrak{s} \varphi(w)$.

Since $\varphi$ is a primitive morphism, its fixed point $\boldsymbol{u}$ is uniformly recurrent, and thus for every $v \in \mathcal{L}(\boldsymbol{u})$ there exists $c \in\{0,1\}$ such that $c v \in \mathcal{L}(\boldsymbol{u})$. We then have $\varphi(c v)=$ $\varphi(c) \varphi(v) \in \mathcal{L}(\boldsymbol{u})$. Moreover, since $\mathfrak{s} \varphi(v)$ is a proper suffix of $\varphi(c) \varphi(v)$ we have $\mathfrak{s} \varphi(v) \in$ $\mathcal{L}(\boldsymbol{u})$.

Therefore the image of an antipalindrome $w \in \mathcal{L}(\boldsymbol{u})$ under the mapping $w \mapsto \mathfrak{s} \varphi(w)$ is a longer antipalindrome in $\mathcal{L}(\boldsymbol{u})$. By the assumption of primitivity of $\varphi$, we have $0,1 \in \mathcal{L}(\boldsymbol{u})$ and thus either 01 or 10 is a factor of $\boldsymbol{u}$. Therefore $\mathcal{L}(\boldsymbol{u})$ contains a nonempty antipalindrome. The statement follows.

We now give a necessary and sufficient condition for a morphism in class $\mathcal{A}_{1}$ to have a fixed point with arbitrarily long palindromes.

Proposition 13. Let $\varphi$ be a morphism in class $\mathcal{A}_{1}$ and let $\boldsymbol{u}$ be its aperiodic fixed point. Then $\boldsymbol{u}$ is palindromic if and only if $\mathfrak{s}=\varepsilon$ and $\mathfrak{p}$ is a palindrome.

Proof. First realize that if $\mathfrak{s}=\varepsilon$ and $\mathfrak{p}$ is a palindrome then by Observation 1, $\mathrm{E}(\mathfrak{p})$ is a palindrome and thus $\varphi$ belongs to class $\mathcal{P}$. Consequently, $\mathcal{L}(\boldsymbol{u})$ contains infinitely many palindromes.

For the opposite implication, let us find $\varphi_{L}$ and $\varphi_{R}$, i.e., the leftmost and rightmost conjugate respectively to $\varphi$. Let $x, y \in\{0,1\}^{*}$ be such that $\mathfrak{p}=x y \mathrm{E}(x)$, where $x$ is the longest possible. That is, either $y=\varepsilon$ or the first and the last letter of $y$ coincide. Therefore $\varphi(0)=x y \mathrm{E}(x) \mathfrak{s}$ and $\varphi(1)=x \mathrm{E}(y) \mathrm{E}(x) \mathfrak{s}$. If $y=\varepsilon$ then $\varphi(0)=\varphi(1)$ and a fixed point of $\varphi$ is periodic, a contradiction. Thus $y \neq \varepsilon$ and

$$
\begin{aligned}
\varphi_{L}(0)=y \mathrm{E}(x) \mathfrak{s} x, & \varphi_{L}(1)=\mathrm{E}(y) \mathrm{E}(x) \mathfrak{s} x, \\
\varphi_{R}(0)=\mathrm{E}(x) \mathfrak{s} x y, & \varphi_{L}(1)=\mathrm{E}(x) \mathfrak{s} x \mathrm{E}(y) .
\end{aligned}
$$

By Proposition 7, $\mathrm{R}\left(\varphi_{L}(0)\right)=\varphi_{R}(0)$, which implies that $y=\mathrm{R}(y), \mathrm{R}(x)=\mathrm{E}(x)$, and $\mathrm{R}(\mathfrak{s})=\mathfrak{s}$. It follows that $\mathfrak{s}=\varepsilon, x=\varepsilon$, and $y$ is a palindrome. Then indeed

$$
\begin{aligned}
& \varphi_{L}(0)=\varphi_{R}(0)=\varphi(0)=y, \\
& \varphi_{L}(1)=\varphi_{R}(1)=\varphi(1)=\mathrm{E}(y) .
\end{aligned}
$$




\subsection{Class $\mathcal{A}_{2}$ - non-uniform morphisms}

The second class of considered morphisms contains morphisms that are non-uniform in general. For its definition we use the Thue-Morse morphism $\Theta$.

Definition 14. A morphism $\psi:\{0,1\}^{*} \rightarrow\{0,1\}^{*}$ is said to be in class $\mathcal{A}_{2}$ if there exist a non-empty word $\mathfrak{w} \in\{0,1\}^{*}$ and $k, h \in \mathbb{N}$ such that

$$
\psi(0)=\Theta\left(\mathfrak{w}(\mathrm{R}(\mathfrak{w}) \mathfrak{w})^{k}\right), \quad \psi(1)=\Theta\left((\mathrm{R}(\mathfrak{w}) \mathfrak{w})^{h} \mathrm{R}(\mathfrak{w})\right) .
$$

Note that a morphism in class $\mathcal{A}_{2}$ is necessarily primitive.

Remark 15. If $\psi$ is in class $\mathcal{A}_{2}$ with $k=h$ then $\psi$ is also in class $\mathcal{A}_{1}$, where $\mathfrak{s}=\varepsilon$ and $\mathfrak{p}=\Theta\left(\mathfrak{w}(\mathrm{R}(\mathfrak{w}) \mathfrak{w})^{k}\right)$. That is a consequence of the relation $\Theta R=E \Theta$ (cf. Observation 1), as $E(\psi(0))=E \Theta\left(\mathfrak{w}(\mathrm{R}(\mathfrak{w}) \mathfrak{w})^{k}\right)=\Theta \mathrm{R}\left(\mathfrak{w}(\mathrm{R}(\mathfrak{w}) \mathfrak{w})^{k}\right)=\Theta\left((\mathrm{R}(\mathfrak{w}) \mathfrak{w})^{k} \mathrm{R}(\mathfrak{w})\right)=\psi(1)$.

Remark 16. One can show that if $\psi$ is in class $\mathcal{A}_{2}$, then $\psi \Theta$ belongs to $\mathcal{E}-\mathcal{P}$. Indeed, $(\psi \Theta)(0)=\psi(01)=\Theta\left((\mathfrak{w} \mathrm{R}(\mathfrak{w}))^{k+h+1}\right)$ and $(\psi \Theta)(1)=\psi(10)=\Theta\left((\mathrm{R}(\mathfrak{w}) \mathfrak{w})^{k+h+1}\right)$. Clearly, both $(\mathfrak{w} R(\mathfrak{w}))^{k+h+1}$ and $(\mathrm{R}(\mathfrak{w}) \mathfrak{w})^{k+h+1}$ are palindromes. Using the fact that $\Theta(v)$ is an antipalindrome if $v$ is a palindrome, we derive that $\psi \Theta$ is of the desired form. The opposite implication is not obvious.

Proposition 17. Let $\boldsymbol{u}$ be a fixed point of a morphism in class $\mathcal{A}_{2}$. Then $\mathcal{L}(\boldsymbol{u})$ contains infinitely many antipalindromes.

We first prove two auxiliary lemmas.

Lemma 18. Let $\psi$ be a morphism in class $\mathcal{A}_{2}$ and let $v \in\{0,1\}^{*}$. Then $\psi(\Theta(\mathrm{R}(v)))=$ $\mathrm{E}(\psi(\Theta(v)))$.

Proof. Since both $\psi \Theta \mathrm{R}$ and $\mathrm{E} \psi \Theta$ are antimorphisms it is enough to prove the formula just for the letters 0,1 . We have

$$
\begin{aligned}
\psi(\Theta(0)) & =\psi(01)=\Theta(\underbrace{(\mathfrak{w R}(\mathfrak{w}))^{k+h+1}}_{\text {palindrome }})=\Theta\left(\mathrm{R}(\mathfrak{w} \mathrm{R}(\mathfrak{w}))^{k+h+1}\right)= \\
& =\mathrm{E} \Theta\left((\mathfrak{w R}(\mathfrak{w}))^{k+h+1}\right)=\mathrm{E}(\psi(01))=\mathrm{E}(\psi(\Theta(0))),
\end{aligned}
$$

and

$$
\begin{aligned}
\psi(\Theta(1)) & =\psi(10)=\Theta(\underbrace{(\mathrm{R}(\mathfrak{w}) \mathfrak{w})^{k+h+1}}_{\text {palindrome }})=\Theta\left(\mathrm{R}(\mathrm{R}(\mathfrak{w}) \mathfrak{w})^{k+h+1}\right)= \\
& =\mathrm{E} \Theta\left((\mathrm{R}(\mathfrak{w}) \mathfrak{w})^{k+h+1}\right)=\mathrm{E}(\psi(10))=\mathrm{E}(\psi(\Theta(1))),
\end{aligned}
$$

where we used the fact that $\Theta \mathrm{R}=\mathrm{E} \Theta$.

Lemma 19. Let $\psi$ be a morphism in class $\mathcal{A}_{2}$ and let $v \in\{0,1\}^{*}$ be such that $\Theta(v)$ is an antipalindrome. Then $\psi(\Theta(v))$ is an antipalindrome.

Proof. Recall from Observation 1 that $\Theta(v)$ is an antipalindrome if and only if $v$ is a palindrome. Then $\mathrm{E}(\psi(\Theta(v)))=\mathrm{E}(\psi(\Theta(\mathrm{R}(v))))=\psi(\Theta(\mathrm{R}(\mathrm{R}(v))))=\psi(\Theta(v))$, where the penultimate equality follows from Lemma 18 . 
Proof of Proposition 17. Obviously, the fixed point $\boldsymbol{u}$ of $\psi$ contains either the antipalindrome 01 or 10. By repeated application of Lemma 19 on it, we get increasingly longer antipalindromes in $\mathcal{L}(\boldsymbol{u})$.

As in the case of morphisms in class $\mathcal{A}_{1}$, we give a necessary and sufficient condition for a morphism in class $\mathcal{A}_{2}$ to have a fixed point with arbitrarily long palindromes.

Proposition 20. Let $\psi$ be a morphism in class $\mathcal{A}_{2}$ and let $\boldsymbol{u}$ be its aperiodic fixed point. Then $\boldsymbol{u}$ is palindromic if and only if $\mathfrak{w}$ from Definition 14 is an antipalindrome.

Proof. If $\mathfrak{w}$ is an antipalindrome, then by Observation 1, $\Theta(\mathfrak{w})$ is a palindrome, and consequently, also $\psi(0)$ and $\psi(1)$ are palindromes. Therefore the morphism $\psi$ belongs to class $\mathcal{P}$, and the language $\mathcal{L}(\boldsymbol{u})$ contains infinitely many palindromes.

Suppose on the other hand that $\boldsymbol{u}$ is palindromic. Let $\mathfrak{w}=x y \mathrm{R}(x)$, for some $x, y \in$ $\{0,1\}^{*}$ and let $x$ be the longest prefix permitting to write $\mathfrak{w}$ in such form. Necessarily, $y \neq \varepsilon$, since otherwise $\psi(0)=\psi(1)$ and $\boldsymbol{u}$ is periodic. Moreover, fst $(y) \neq \operatorname{lst}(y)$. Therefore for the leftmost and rightmost conjugate to $\psi$ we have

$$
\begin{aligned}
& \psi_{L}(0)=\Theta\left(y \mathrm{R}(x)(x \mathrm{R}(y) \mathrm{R}(x) x y \mathrm{R}(x))^{k}\right) \Theta(x), \\
& \psi_{L}(1)=\Theta\left(\mathrm{R}(y) \operatorname{R}(x)(x y \operatorname{R}(x) x \operatorname{R}(y) \mathrm{R}(x))^{h}\right) \Theta(x), \\
& \psi_{R}(0)=\Theta(\mathrm{R}(x)) \Theta\left((x y \operatorname{R}(x) x \operatorname{R}(y) \mathrm{R}(x))^{k} x y\right), \\
& \psi_{R}(1)=\Theta(\mathrm{R}(x)) \Theta\left((x \mathrm{R}(y) \mathrm{R}(x) x y \mathrm{R}(x))^{h} x \mathrm{R}(y)\right) .
\end{aligned}
$$

Since $\boldsymbol{u}$ is palindromic, by Proposition 7, we have $\mathrm{R}\left(\psi_{L}(a)\right)=\psi_{R}(a)$ for $a=0,1$. Comparing with the above we obtain that $\mathrm{R} \Theta(x)=\Theta \mathrm{R}(x)=\mathrm{E} \Theta(x)$ and thus $x=\varepsilon$. Further, we have $\operatorname{R} \Theta(y)=\Theta(y)$, which means that $\Theta(y)$ is a palindrome and thus (again by Observation 1) $y=\mathfrak{w}$ is an antipalindrome.

The following proposition shows that changing the parameters $k, h$ in the definition of a morphism $\psi$ in class $\mathcal{A}_{2}$ while keeping their sum $k+h$ fixed does not change the fixed points.

Proposition 21. Let $\psi$ be a morphism in class $\mathcal{A}_{2}$ with $k+h \geq 1$. Let $\xi:\{0,1\}^{*} \rightarrow$ $\{0,1\}^{*}$ be given by

$$
\xi(0)=\Theta(\mathfrak{w}) \quad \text { and } \quad \xi(1)=\Theta\left((\mathrm{R}(\mathfrak{w}) \mathfrak{w})^{k+h} \mathrm{R}(\mathfrak{w})\right) .
$$

Then $\boldsymbol{u}$ is a fixed point of $\psi$ if and only if it is a fixed point of $\xi$.

Proof. Note that

$$
\begin{aligned}
& \xi(\Theta(0))=\xi(01)=\psi(01)=\psi(\Theta(0)) \\
& \xi(\Theta(1))=\xi(10)=\psi(10)=\psi(\Theta(1)),
\end{aligned}
$$

and thus

$$
\xi \Theta=\psi \Theta .
$$

Since $\boldsymbol{u}=\psi(\boldsymbol{u})$ there is an infinite word $\boldsymbol{v}$ such that $\boldsymbol{u}=\Theta(\boldsymbol{v})$. Using (4) we get

$$
\xi(\boldsymbol{u})=\xi(\Theta(\boldsymbol{v}))=\psi(\Theta(\boldsymbol{v}))=\psi(\boldsymbol{u})=\boldsymbol{u} .
$$

The argumentation for the opposite implication is the same, interchanging the role of $\xi$ and $\psi$. 
Remark 22. Proposition 21 implies that a morphism $\xi$ in class $\mathcal{A}_{2}$ with $k+h \geq 1$ is not rigid. On the other hand, if $\psi$ is in class $\mathcal{A}_{2}$ with $k+h=0$ and $\mathfrak{w}=01$ then $\psi=\Theta^{2}$ is an iterate of the Thue-Morse morphism $\Theta$. It is shown in [18] that its fixed points are rigid. For $k+h=0$ and $\mathfrak{w} \neq 01$, the situation is not clear.

\section{Palindromes and antipalindromes in periodic words}

Let us first study properties of eventually periodic words which contain arbitrarily long antipalindromes. The statements we show here are direct analogues of similar facts for palindromes, namely Propositions 23 and 24 which we cite from [4, 1].

Proposition 23 ([4]). Let $\boldsymbol{u}=v w^{\infty}$, where $w \neq \varepsilon$ be an eventually periodic palindromic word. Then $w=p q$ where $p, q$ are palindromes.

Proposition 24 ([1]). Let $\boldsymbol{u}$ be an eventually periodic palindromic word. If $\boldsymbol{u}$ is recurrent then $\boldsymbol{u}$ is a fixed point of a morphism in class $\mathcal{P}$.

We prove the analogues of the above two results for antipalindromic words as Lemmas 25 and 26. Lemma 25 has been also proven in [13].

Lemma 25. Let $\boldsymbol{u}=v w^{\infty}$, where $w \neq \varepsilon$, be an eventually periodic antipalindromic word. Then $w=w_{1} w_{2}$ where $w_{1}, w_{2}$ are antipalindromes.

Proof. Since $\mathcal{L}(\boldsymbol{u})$ contains infinitely many antipalindromes it necessarily contains antipalindromes in the form $b w^{k} c$, where $k \geq 1$, and $b$ and $c$ is a proper suffix and a proper prefix of $w$, respectively. Without loss of generality we can assume that $|b| \leq|c|<|w|$. Let $w_{1}$ be the prefix of $c$ of length $|c|-|b|$. Then $w^{k} w_{1}$ is also an antipalindrome. Let us denote $w=w_{1} w_{2}$. We have

$$
\left(w_{1} w_{2}\right)^{k} w_{1}=\mathrm{E}\left(\left(w_{1} w_{2}\right)^{k} w_{1}\right)=\mathrm{E}\left(w_{1}\right)\left(\mathrm{E}\left(w_{2}\right) \mathrm{E}\left(w_{1}\right)\right)^{k} .
$$

Thus $w_{1}=\mathrm{E}\left(w_{1}\right)$ and $w_{2}=\mathrm{E}\left(w_{2}\right)$.

Lemma 26. Let $\boldsymbol{u}$ be an eventually periodic antipalindromic word. If $\boldsymbol{u}$ is recurrent then it is a fixed point of a morphism in class $\mathcal{A}_{1}$.

Proof. A recurrent eventually periodic word is necessarily periodic, i.e., $\boldsymbol{u}=w^{\infty}$, where $w=w_{1} w_{2}$ by Lemma 25. The word $\boldsymbol{u}$ is obviously fixed by the morphism given by $0 \mapsto w$ and $1 \mapsto w$, which is in class $\mathcal{A}_{1}\left(\mathfrak{p}=w_{1}, \mathfrak{s}=w_{2}\right)$.

The following theorem puts together the above facts and gives a description of eventually periodic words which are both palindromic and antipalindromic.

Proposition 27. Let $\boldsymbol{u}$ be an eventually periodic word which contains infinitely many palindromes and antipalindromes. Then there exist a word $b \in\{0,1\}^{*}$ and a palindrome $c \in\{0,1\}^{*}$ such that $\boldsymbol{u}=b(c \mathrm{E}(c))^{\infty}$.

Proof. If $\boldsymbol{u}=v w^{\infty}$, where $w \neq \varepsilon$, then by Lemma 25 we have $w=w^{\prime} w^{\prime \prime}$, where $w^{\prime}, w^{\prime \prime}$ are antipalindromes. Thus $w^{\prime}=\mathrm{E}(f) f$ for some $f$ and $\boldsymbol{u}=v \mathrm{E}(f)\left(f w^{\prime \prime} \mathrm{E}(f)\right)^{\infty}$. Therefore $\boldsymbol{u}$ is of the form $\boldsymbol{u}=b a^{\infty}$ where $a=f w^{\prime \prime} \mathrm{E}(f)$ is an antipalindrome. By Proposition 23 $a$ can be written as a concatenation of two palindromes $a=p q$. Using item (iii) of Proposition 2 we infer that there is a palindrome $c$ such that $a=(c \mathrm{E}(c))^{k}$ for some $k \in \mathbb{N}$. 


\section{Languages of aperiodic words containing infinitely many antipalindromes}

In this section we will demonstrate several properties of aperiodic antipalindromic words which will be used in the proof of our main results. Lemmas 28, 29 and 31 are analogues of similar statements for palindromic words.

Lemma 28. Let $\boldsymbol{u}$ be a uniformly recurrent antipalindromic word. Then $\mathcal{L}(\boldsymbol{u})$ is closed under the antimorphism $\mathrm{E}$.

Proof. Since $\boldsymbol{u}$ is uniformly recurrent there is a function $r: \mathbb{N} \rightarrow \mathbb{N}$ such that every factor of $\boldsymbol{u}$ of length $r(n)$ contains all the factors of $\boldsymbol{u}$ of length $n$. Let $w \in \mathcal{L}(\boldsymbol{u})$ and let $v \in \mathcal{L}(w)$ be an antipalindrome such that $|v| \geq r(|w|)$. Then both $w$ and $\mathrm{E}(w)$ are factors of $\boldsymbol{u}$.

Lemma 29. Let $\boldsymbol{u}$ be an infinite antipalindromic word. Then there is an infinite word $\boldsymbol{v}$ such that $\mathrm{E}(v) v \in \mathcal{L}(\boldsymbol{u})$ for every prefix $v$ of $\boldsymbol{v}$.

Proof. Every antipalindrome is of the form $\mathrm{E}(w) w$. Since $\boldsymbol{u}$ contains infinitely many antipalindromes, there are an infinite number of words $w$ such that $\mathrm{E}(w) w \in \mathcal{L}(\boldsymbol{u})$. Define $\boldsymbol{v}=v_{1} v_{2} v_{3} \cdots$ in the following way: let $v_{1}$ be a letter which is a prefix of infinitely many $w$ such that $\mathrm{E}(w) w \in \mathcal{L}(\boldsymbol{u})$; let $v_{2}$ be a letter for which $v_{1} v_{2}$ is a prefix of infinitely many $w$ such that $\mathrm{E}(w) w \in \mathcal{L}(\boldsymbol{u})$; etc.

Remark 30. If $\boldsymbol{u}$ is uniformly recurrent then the languages of $\boldsymbol{u}$ and of the (both-sided) infinite word $\mathrm{E}(\boldsymbol{v}) \boldsymbol{v}$ coincide. Otherwise, we have $\mathcal{L}(\mathrm{E}(\boldsymbol{v}) \boldsymbol{v}) \subset \mathcal{L}(\boldsymbol{u})$.

The following lemma is an analogy of Lemma 25 of [14 where the authors study words containing infinitely many palindromes.

Lemma 31. Let $\boldsymbol{u}$ be an aperiodic uniformly recurrent antipalindromic word. Then $\mathcal{L}(\boldsymbol{u})$ contains infinitely many antipalindromic bispecial factors.

Proof. We use the fact that every factor $f$ of an aperiodic word $\boldsymbol{u}$ which is not itself right special has unique minimal (right) prolongation into a right special factor of $\boldsymbol{u}$, i.e., there is a unique word $e$ such that $f e$ is a right special in $\mathcal{L}(\boldsymbol{u})$ while $f e^{\prime}$ is not right special for each proper prefix $e^{\prime}$ of $e$. An analogous claim holds also for left special factors.

For each $N \in \mathbb{N}$ we demonstrate how to construct an antipalindromic bispecial factor in $\mathcal{L}(\boldsymbol{u})$ of length greater than or equal to $N$. Let $w$ be a prefix of the infinite word $\boldsymbol{v}$ from Lemma 29 (recall that $\mathrm{E}(w) w \in \mathcal{L}(\boldsymbol{u}))$ such that $|\mathrm{E}(w) w| \geq N$. If $\mathrm{E}(w) w$ is right special then it is left special too, since by Lemma 28 the language $\mathcal{L}(\boldsymbol{u})$ is closed under E. Let $\mathrm{E}(w) w$ be not a right special factor. Then there exists $e$ such that $\mathrm{E}(w) w e$ is right special. Recall that for every proper prefix $e^{\prime}$ of $e$ the factor $\mathrm{E}(w) w e^{\prime}$ has unique right extension, thus we is also a prefix of $\boldsymbol{v}$.

Since $\mathcal{L}(\boldsymbol{u})$ is closed under $\mathrm{E}, \mathrm{E}(e) \mathrm{E}(w) w$ is unique left prolongation of $\mathrm{E}(w) w$, and, moreover, it is a left special factor. Therefore the word $\mathrm{E}(e) \mathrm{E}(w) w e$ is a bispecial factor in $\mathcal{L}(\boldsymbol{u})$.

The last lemma of this section speaks about frequencies of letters in an antipalindromic word. This result has no counterpart in palindromic words. Here we assume that such frequencies exist, which is not necessarily true even for uniformly recurrent words. Later on, this assumption will be ensured by taking fixed points of primitive morphisms. 
Lemma 32. Let $\boldsymbol{u}$ be a binary infinite word containing infinitely many antipalindromes. Suppose that the frequency of a letter a in $\boldsymbol{u}$ exists, namely

$$
\varrho(a)=\lim _{|w| \rightarrow \infty} \frac{|w|_{a}}{|w|} .
$$

Then the frequency of both letters in $\boldsymbol{u}$ is equal to $1 / 2$.

Proof. Since the frequency of a letter $a$ in $\boldsymbol{u}$ exists, it can be computed as corresponding limit of any subsequence of factors of $\boldsymbol{u}$. Let us consider a subsequence obtained by taking only antipalindromic factors $w$ of $\boldsymbol{u}$. As $\mathrm{E}(w)=w$, we have

$$
\frac{|w|_{a}}{|w|}=\frac{1}{2} \quad \text { for } a=0,1
$$

Therefore $\varrho(0)=\frac{1}{2}=\varrho(1)$.

\section{Antipalindromes in fixed points of morphisms}

In this section we present our main results that concern antipalindromic fixed points of primitive morphisms. The case of periodic words is covered by Lemma 26, thus we focus on aperiodic fixed points.

The languages of infinite words fixed by mutually conjugated primitive morphisms coincide, cf. item (iii) of Proposition 2, For a chosen morphism $\varphi$ with an aperiodic antipalindromic fixed point we consider the rightmost and leftmost conjugate $\varphi_{R}, \varphi_{L}$ and the relation between them, namely the conjugacy word $q \in\{0,1\}^{*}$ such that

$$
q \varphi_{L}(w)=\varphi_{R}(w) q \quad \text { for every } w \in\{0,1\}^{*} .
$$

Crucial for the demonstration of our results is the description of antipalindromic bispecial factors in fixed points of primitive morphisms derived in the sense of Theorem 8 . The following lemma testifies about them and the conjugacy word $q$ of (5).

Lemma 33. Let $\varphi$ be a primitive binary morphism with an aperiodic antipalindromic fixed point $\boldsymbol{u}$. Then the word $q$ from (5) is an antipalindrome and $\mathcal{L}(\boldsymbol{u})$ contains infinitely many antipalindromes of the form $\varphi_{R}(w) q$ for some $w \in \mathcal{L}(\boldsymbol{u})$.

If, moreover, $\boldsymbol{u}$ contains infinitely many palindromes, then $q=\varepsilon$ and $\mathcal{L}(\boldsymbol{u})$ contains infinitely many antipalindromes of the form $\varphi(w)$ for some $w \in \mathcal{L}(\boldsymbol{u})$.

Proof. By Lemma 31 the language $\mathcal{L}(\boldsymbol{u})$ contains infinitely many antipalindromic bispecial factors. By Theorem 8, any sufficiently long bispecial factor is of the form $\varphi_{R}(w) q$, where $w \in \mathcal{L}(\boldsymbol{u})$. Consider $w$ such that $\varphi_{R}(w) q$ is an antipalindrome. Using (5) we obtain

$$
q \varphi_{L}(w)=\varphi_{R}(w) q=\mathrm{E}\left(\varphi_{R}(w) q\right)=\mathrm{E}(q) \mathrm{E}\left(\varphi_{R}(w)\right)
$$

Therefore $q=\mathrm{E}(q)$.

If $\boldsymbol{u}$ contains also infinitely many palindromes, by Lemma 20 from [14], $q$ is a palindrome. Altogether, $q=\varepsilon$ and consequently $\varphi_{R}=\varphi$. Thus $\varphi_{R}(w) q=\varphi(w)$.

We are now in position to state our main results. They are separated into two theorems. The first one of them, Theorem 34, states for uniform morphisms the opposite of Proposition 11 . 
Theorem 34. Let $\boldsymbol{u}$ be an aperiodic fixed point of a primitive binary uniform morphism $\varphi$ such that $\boldsymbol{u}$ contains infinitely many antipalindromes. Then $\varphi$ or $\varphi^{2}$ is conjugated to a morphism in class $\mathcal{A}_{1}$.

Proof. Since $\varphi$ has an aperiodic fixed point, it is acyclic. Therefore the leftmost and rightmost conjugates $\varphi_{L}, \varphi_{R}$ to the morphism $\varphi$ exist and $\varphi$ is marked. Without loss of generality we can assume for the first and last letters that

$$
\begin{array}{ll}
\operatorname{fst}\left(\varphi_{L}(a)\right)=a & \text { for } a=0,1, \\
\operatorname{lst}\left(\varphi_{R}(a)\right)=a & \text { for } a=0,1 .
\end{array}
$$

Otherwise, we take $\varphi^{2}$ instead of $\varphi$.

Let $w=w_{1} w_{2} \cdots w_{n}$ be a factor such that $\varphi_{R}(w) q$ is an antipalindrome, which exists by Lemma 33 . Then, similarly as in (6) we have

$$
\varphi_{L}\left(w_{1}\right) \varphi_{L}\left(w_{2}\right) \cdots \varphi_{L}\left(w_{n}\right)=\mathrm{E}\left(\varphi_{R}\left(w_{n}\right)\right) \mathrm{E}\left(\varphi_{R}\left(w_{n-1}\right)\right) \cdots \mathrm{E}\left(\varphi_{R}\left(w_{1}\right)\right) .
$$

From the equation (7) and from the property $|\varphi(0)|=|\varphi(1)|$ we see that

$$
w_{1}=\mathrm{E}\left(w_{n}\right), w_{2}=\mathrm{E}\left(w_{n-1}\right), \ldots
$$

and, consequently, for every letter $a$ we have

$$
\varphi_{L}(\mathrm{E}(a))=\mathrm{E}\left(\varphi_{R}(a)\right)
$$

There are two different cases based on the form of the conjugacy word $q$ from (5). If $q$ is empty then $\varphi_{L}=\varphi_{R}=\varphi$, by $(8)$ it fulfills $\varphi(1)=\mathrm{E}(\varphi(0))$, and, therefore, $\varphi$ is in class $\mathcal{A}_{1}$.

Let $q \neq \varepsilon$. Combining (5) and (8) we get

$$
\varphi_{R}(1) q=q \varphi_{L}(1)=q \mathrm{E}\left(\varphi_{R}(0)\right) .
$$

Let us denote $x:=\varphi_{R}(1)$ and $y:=\mathrm{E}\left(\varphi_{R}(0)\right)$. Then the formula $(9)$ can be seen as an equation on words of the form $x q=q y$. Thus by item (ii) of Proposition 2, there exist $e, f \in\{0,1\}^{*}$ and $i \in \mathbb{N}$ such that

$$
x=e f, \quad y=f e, \quad q=(e f)^{i} e .
$$

At first, let us inspect the case where $i=0$. Since by Lemma 33 the word $q=e$ is an antipalindrome, we can write

$$
\varphi_{R}(1)=e f \quad \text { and } \quad \varphi_{R}(0)=\mathrm{E}(y)=\mathrm{E}(f e)=e \mathrm{E}(f)
$$

and, therefore, $\varphi$ is conjugated to a morphism in class $\mathcal{A}_{1}$.

Now, let $i \geq 1$. Since $q=(e f)^{i} e$ is an antipalindrome, we have that $e, f$ are antipalindromes, too. This implies that $\varphi_{R}(1)=e f=\mathrm{E}(f e)=\varphi_{R}(0)$, i.e., the fixed point is periodic. This is a contradiction, and, therefore, the case $i \geq 1$ will not occur.

As an application of Theorem 34 we will show an example of a uniform morphism in class $\mathcal{E}$ - $\mathcal{P}$ which does not generate a palindromic fixed point. 
Example 35. Consider the binary morphism $\varphi$ defined by $\varphi(0)=0101$ and $\varphi(1)=1100$. Since both $\varphi(0)$ and $\varphi(1)$ are antipalindromes, $\varphi$ belongs to class $\mathcal{E}-\mathcal{P}$. At the same time, one can check that neither $\varphi$ nor $\varphi^{2}$ belongs to $\mathcal{A}_{1}$. Therefore by Theorem 34 , the fixed point of $\varphi$ contains only finitely many antipalindromes.

In the second main theorem, we show that only morphisms of class $\mathcal{A}_{2}$ have aperiodic fixed points containing infinitely many of both antipalindromes and palindromes. Even with the additional requirement of palindromicity of $\boldsymbol{u}$, the proof of Theorem 36 is complicated. It is likely that the demonstration of the fact that any non-uniform morphism generating aperiodic infinite antipalindromic words is in class $\mathcal{A}_{2}$ would require a novel approach using new techniques.

Theorem 36. Let $\boldsymbol{u}$ be an aperiodic fixed point of a primitive binary non-uniform morphism $\varphi$ such that $\mathcal{L}(\boldsymbol{u})$ contains an infinite number of palindromes as well as antipalindromes. Then either $\varphi$ or $\varphi^{2}$ is a morphism in class $\mathcal{A}_{2}$ with $\mathfrak{w}$ being an antipalindrome.

For the proof of Theorem 36 we need, besides the properties of languages of antipalindromic words demonstrated in Section 5, also a number of auxiliary statements. We collect them together with the proof of Theorem 36 in the following section.

\section{Proof of Theorem 36}

Throughout this section we will work only with morphisms of a special form. Lemma 37 then shows that such a restriction is justified by assumptions of Theorem 36 .

Condition $(\star)$ : We say that $\varphi$ and $\boldsymbol{u}$ satisfy condition $(\star)$ if $\varphi$ is a primitive binary non-uniform morphism such that $\varphi(a)$ is a palindrome with prefix $a$ for $a=0,1$, and that $\boldsymbol{u}$ is its aperiodic antipalindromic fixed point.

Lemma 37. Let $\boldsymbol{u}$ be an aperiodic fixed point of a primitive binary non-uniform morphism $\varphi$ such that $\mathcal{L}(\boldsymbol{u})$ contains an infinite number of palindromes as well as antipalindromes. Then $\boldsymbol{u}$ with either $\varphi$ or $\varphi^{2}$ satisfy condition $(\star)$.

Proof. Since $\boldsymbol{u}$ is palindromic, it follows from a result by Tan [25] that $\varphi$ or $\varphi^{2}$ is a conjugate to $\xi$ from class $\mathcal{P}$, i.e., $\xi(0)=p_{0} p$ and $\xi(1)=p_{1} p$, where $p_{0}, p_{1}, p$ are palindromes. Since $\xi$ is also a primitive morphism, the language of its fixed point coincides with $\mathcal{L}(\boldsymbol{u})$. By Lemma 33, the conjugacy word $q$ between morphism $\xi_{R}, \xi_{L}$ is empty, and thus we have $\xi=\xi_{R}=\xi_{L}$. Therefore there is no other morphism conjugated with $\xi$, and thus either $\varphi$ or $\varphi^{2}$ is equal to $\xi$, and, moreover, the palindrome $p$ is empty. This means that either $\xi=\varphi$ or $\xi=\varphi^{2}$ together with $\boldsymbol{u}$ satisfy condition $(\star)$.

The above lemma shows that under the assumptions of Theorem 36, the words $\varphi(0)$, $\varphi(1)$ are palindromes. In order to complete the proof of Theorem 36, we need to show that these palindromes are of a very special form given by Definition 14 . The demonstration needs two technical statements, which follow as Lemmas 40 and 41 . For the sake of lucidity, we have separated parts of the proof of Lemma 40 into two auxiliary facts, formulated as Lemmas 38 and 39 ,

Lemma 38. Let $\varphi$ and $\boldsymbol{u}$ satisfy condition $(\star)$. Suppose that there exists a nonempty word $x \in\{0,1\}^{*}$ such that $x \neq \mathrm{E}(x)$ and $\varphi(0), \varphi(1) \in\{x, \mathrm{E}(x)\}^{*}$. For $a=0,1$ denote by $k_{a}, h_{a}$ the number of words $x, \mathrm{E}(x)$, respectively, in $\varphi(a)$. Then $k_{0}+k_{1}=h_{0}+h_{1}$. 
Proof. We shall prove the statement by contradiction. Suppose that $\mathcal{L}(\boldsymbol{u})$ contains infinitely many antipalindromes and $k_{0}+k_{1} \neq h_{0}+h_{1}$. Then at least one of $k_{0}-h_{0}$, $k_{1}-h_{1}$ is non-zero. Assume without loss of generality that $k_{0} \neq h_{0}$. Then $\frac{h_{1}-k_{1}}{k_{0}-h_{0}} \neq 1$ and there exists $\delta>0$ such that $\frac{h_{1}-k_{1}}{k_{0}-h_{0}} \notin(1-\delta, 1+\delta)$. As $\boldsymbol{u}$ is a fixed point of a primitive morphism, the frequencies of letters exist [21. By Lemma 32 the frequencies of both letters in $\boldsymbol{u}$ are equal to $\frac{1}{2}$, thus there is $N \in \mathbb{N}$ such that for every factor $w$ of $\boldsymbol{u}$ of the length $|w|>N$ we have $\frac{|w|_{0}}{|w|_{1}} \in(1-\delta, 1+\delta)$. Choose a factor $w$ such that $\varphi(w)$ is an antipalindrome in $\boldsymbol{u}$ of length greater than $N(|\varphi(0)|+|\varphi(1)|)$. Such $w \in \mathcal{L}(\boldsymbol{u})$ exists by Lemma 33. Evidently, $|w|>N$. By assumption, $\varphi(w)$ is a concatenation of words $x$ and $\mathrm{E}(x)$, where $|x|=|\mathrm{E}(x)|$ and $x \neq \mathrm{E}(x)$. Therefore $\varphi(x)$ has to consist of the same number of factors $x$ and $\mathrm{E}(x)$, that is, $k_{0}|w|_{0}+k_{1}|w|_{1}=h_{0}|w|_{0}+h_{1}|w|_{1}$. Consequently,

$$
\frac{|w|_{0}}{|w|_{1}}=\frac{h_{1}-k_{1}}{k_{0}-h_{0}} \notin(1-\delta, 1+\delta) \text {. }
$$

Note that in the proof of Lemma 38 we have not used that $\varphi(0), \varphi(1)$ are palindromes.

Lemma 39. Let $c$ be a nonempty palindrome, and let $N \geq 1$. Then $(\mathrm{E}(c) c)^{N} \mathrm{E}(c)$ is a primitive word.

Proof. We shall prove by contradiction. Let $n \in \mathbb{N}, n \geq 2$ be the largest integer such that $z^{n}=(\mathrm{E}(c) c)^{N} \mathrm{E}(c)$ for some $z \in\{0,1\}^{*}$, and let $w:=z^{n}$. As $\mathrm{E}(c)$ is a palindrome, both $z$ and $w$ are palindromes too. Obviously, the word $w$ has periods $|\mathrm{E}(c) c|=2|c|$ and $|z|$.

i) At first, assume that $n+N \geq 4$. Thus either $N \geq 2$, or $N=1$ and $n \geq 3$. By simple inspection, we derive from $|w|=(2 N+1)|c|=n|z|$ that $|w| \geq 2|c|+|z|$. By Theorem 3, $w$ has also the period $\ell=\operatorname{gcd}(|z|, 2|c|)$. If $\ell<|z|$ then $|z|=\ell j$ for some $j \in \mathbb{N}, j \geq 2$ and therefore $z=y^{j}$ for some $y \in\{0,1\}^{*}$, a contradiction with the maximality of $n$. This implies $\operatorname{gcd}(|z|, 2|c|)=|z|$, thus the period $|z|$ of $w$ divides $2|c|$, and there exists $s \in \mathbb{N}$ such that $z^{s}=\mathrm{E}(c) c$. As $z$ is a palindrome it follows that $z^{s}=\mathrm{E}(c) c$ is a palindrome, too, that is,

$$
\mathrm{E}(c) c=\mathrm{R}(\mathrm{E}(c) c)=c \mathrm{E}(c),
$$

where we used the fact that $c$ is a palindrome. By (11) we have $c=\mathrm{E}(c)$. The only word which is a palindrome and an antipalindrome at the same time is $c=\varepsilon$, a contradiction.

ii) Now, assume that $n+N=3$, i.e., $n=2, N=1$, and

$$
z z=\mathrm{E}(c) c \mathrm{E}(c) .
$$

This means that the length of $c$ is even and we can write $c=e f$, where $|e|=|f|=$ $\frac{1}{2}|c|$. Substituting $c=e f$ in 12 we have

$$
z z=\mathrm{E}(e f) e f \mathrm{E}(e f)=\mathrm{E}(f) \mathrm{E}(e) e f \mathrm{E}(f) \mathrm{E}(e),
$$

and since the first and the second half of the word have to be equal we obtain $\mathrm{E}(f)=f, \mathrm{E}(e)=\mathrm{E}(f)$ and $e=\mathrm{E}(e)$. Therefore $z=f f f$, a contradiction with the maximality of $n$. 
Lemma 40. Let $\varphi$ and $\boldsymbol{u}$ satisfy condition $(\star)$. Then

i) there exists a palindrome $c \in\{0,1\}^{*}$ containing 00 or 11 such that

$$
\varphi(0)=(\mathrm{E}(c) c)^{l_{0}} \mathrm{E}(c) \quad \text { and } \quad \varphi(1)=(c \mathrm{E}(c))^{l_{1}} c,
$$

for some $l_{0}, l_{1} \in \mathbb{N}$;

ii) if $w \in \mathcal{L}(\boldsymbol{u})$ is such that $\varphi(w)$ is an antipalindrome, then there exists a palindrome $u$ such that $w=\Theta(u)$;

iii) every $v \in \mathcal{L}(\boldsymbol{u})$ can be expressed in the form $v=x \Theta(z) y$, where $x, y \in\{0,1, \varepsilon\}$, $z \in\{0,1\}^{*}$. Moreover, if either 00 or 11 is a factor of $v$ then $x, y, z$ are uniquely determined.

Proof. We start with the proof of the statement (i). Denote $p_{0}=\varphi(0), p_{1}=\varphi(1)$, without loss of generality we can assume $\left|p_{0}\right|<\left|p_{1}\right|$. Consider a factor $w \in \mathcal{L}(\boldsymbol{u})$ such that $\varphi(w)$ is an antipalindrome, which exists by Lemma 33

At first, let us inspect the case where 0 is a prefix of $\varphi(w)$. As $\varphi(w)$ is an antipalindrome, it necessarily has a suffix 1. It follows from the form of $\varphi$ that $w$ has a prefix 0 and a suffix 1. Let $k \in \mathbb{N}$ be a positive integer such that $w$ has a prefix $0^{k} 1$.

Case 1) $\exists i \in \mathbb{N}, 1 \leq i \leq k$ such that $\left|p_{0}^{i}\right| \geq\left|\mathrm{E}\left(p_{1}\right)\right|>\left|p_{0}^{i-1}\right|$. Since $\left|p_{0}\right|<\left|p_{1}\right|$ we have $i \geq 2$. Recall that $\mathrm{E}(\varphi(w))=\varphi(w)$, the situation is as follows

$$
\begin{aligned}
\varphi(w) & =\overbrace{p_{0} \quad p_{0}}^{i \text {-times }} \cdots p_{0} \\
\mathrm{E}(\varphi(w)) & =\overbrace{\mathrm{E}^{2}\left(p_{1}\right)}^{i} \ldots
\end{aligned}
$$

Thus there exist $p^{\prime}, p^{\prime \prime}$ such that $p^{\prime} \neq \varepsilon, p_{0}=p^{\prime} p^{\prime \prime}$, and $\mathrm{E}\left(p_{1}\right)=\left(p^{\prime} p^{\prime \prime}\right)^{i-1} p^{\prime}$. Since $i \geq 2$ and $\mathrm{E}\left(p_{1}\right)$ is a palindrome, we have that $p^{\prime}$ and $p^{\prime \prime}$ are palindromes. It follows that $p_{0}=p^{\prime} p^{\prime \prime}$ is a palindrome too, and therefore $p^{\prime}$ and $p^{\prime \prime}$ commute. Indeed, $p^{\prime} p^{\prime \prime}=$ $p_{0}=\mathrm{R}\left(p_{0}\right)=p^{\prime \prime} p^{\prime}$. By item (ii) of Proposition 2, this means that there is a palindrome $x$ such that $p^{\prime}=x^{t}$ and $p^{\prime \prime}=x^{s}$ for some $t, s \in \mathbb{N}, t \geq 1$. Consequently, $\varphi$ is of the form $\varphi(0)=x^{t+s}$ and $\mathrm{E}(\varphi(1))=x^{(t+s)(i-1)+t}$. Since $\mathcal{L}(\boldsymbol{u})$ contains infinitely many palindromes, by Lemma 38 , we have $t+s=(t+s)(i-1)+t$, i.e., $0=(t+s)(i-2)+t \geq t \geq 1$, a contradiction. The Case 1) cannot occur.

Case 2) $\left|p_{0}^{k}\right|<\left|\mathrm{E}\left(p_{1}\right)\right|$. The situation is as follows

$$
\begin{aligned}
\varphi(w) & =\overbrace{p_{0}\left[p_{0}\right.}^{k} \cdots p_{0} \\
\mathrm{E}(\varphi(w)) & =\overbrace{\overbrace{\mathrm{E}\left(p_{1}\right)}^{k \text {-times }} \ldots} \cdots
\end{aligned}
$$

Thus there exists $q \in\{0,1\}^{*}$ such that $p_{0}^{k} q=\mathrm{E}\left(p_{1}\right)$, i.e., $p_{1}=\mathrm{E}(q)\left(\mathrm{E}\left(p_{0}\right)\right)^{k}$. As $q$ is also a prefix of $p_{1}$, we derive $q=\mathrm{E}(q)$, i.e., $q$ is an antipalindrome. Since $\mathrm{E}\left(p_{1}\right)$ is a palindrome, we have $p_{0}^{k} q=\mathrm{R}(q) p_{0}^{k}$. By item (ii) of Proposition 2, there exist $A, B \in\{0,1\}^{*}$ and $N \in \mathbb{N}$ such that

$$
q=B A, \quad \mathrm{R}(q)=A B, \quad p_{0}^{k}=(A B)^{N} A .
$$

First two formulae used together, $A B=\mathrm{R}(q)=\mathrm{R}(B A)=\mathrm{R}(A) \mathrm{R}(B)$, imply that $A$ and $B$ are palindromes. Since $q=B A$ is an antipalindrome, we can use item (iii) of 
Proposition 2 to infer that there exists $c \in\{0,1\}^{*}, c$ a palindrome, such that $B=$ $c(\mathrm{E}(c) c)^{s}$ and $A=(\mathrm{E}(c) c)^{t} \mathrm{E}(c)$ for some $s, t \in \mathbb{N}$. Substituting these forms back to 14 we obtain

$$
\begin{aligned}
& p_{0}^{k}=(\mathrm{E}(c) c)^{(t+s+1) N+t} \mathrm{E}(c), \\
& p_{1}=q\left(\mathrm{E}\left(p_{0}\right)\right)^{k}=(c \mathrm{E}(c))^{(t+s+1)(N+1)+t} c .
\end{aligned}
$$

If $(t+s+1) N+t \geq 1$ then by Lemma $39 p_{0}^{k}$ is a primitive word, and, thus $k=1$. Consequently, $w$ has a prefix $0^{k} 1=01$ and the forms of $\varphi(0)$ and $\varphi(1)$ given by (15) are in agreement with the statement of the lemma. On the other hand, if $(t+s+1) N+t=0$ then $N=t=0$. It follows from (15) that $p_{0}^{k}=\mathrm{E}(c)$ and thus $c=d^{k}$ for some palindrome $d$, i.e., $p_{0}=\mathrm{E}(d)$ and $p_{1}=\left(d^{k} \mathrm{E}(d)^{k}\right)^{s+1} d^{k}$. Using Lemma 38 with $x=d, k_{0}=0, h_{0}=1$, $k_{1}=k(s+2)$ and $h_{1}=k(s+1)$, the equality $k_{0}+k_{1}=h_{0}+h_{1}$ implies that $k=1$. Therefore the forms of $\varphi(0)$ and $\varphi(1)$ are in the agreement with the statement of the lemma.

Now consider the case when 1 is a prefix of $\varphi(w)$. Then 0 is a suffix of $\varphi(w)$, and thus 1 is a prefix of $w$ and 0 is a suffix of $w$. The discussion would follow analogically to the case where 0 is a prefix of $\varphi(0)$, with the only exception that we would need to show that if $10^{k}$ is a suffix of $w$ then $k=1$.

Finally, assume that neither 00 nor 11 is a factor of $c$. Then the palindrome $c$, which is a prefix of $\varphi(1)$ and starts with the letter 1 , is of the form $c=(10)^{m} 1$ for some $m$. Thus $\varphi(0)=(01)^{i} 0$ and $\varphi(1)=(10)^{j} 1$ for some $i, j \in \mathbb{N}$. In such a case the fixed point of $\varphi$ is periodic, which is a contradiction with the assuption $(\star)$.

Let us now prove the statement (ii). Note that in the proof of (i) we have shown that if 0 is a prefix of $\varphi(w)$ then $w$ has a prefix 01 and a suffix 1 , and $p_{0}$ and $p_{1}$ have the form (13). We will now verify that the penultimate letter $a$ in $w$ is equal to 0 , i.e., that $w$ has 01 as a suffix. The situation is as follows

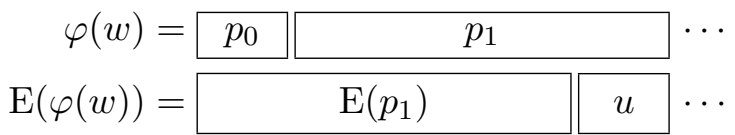

where $u$ is a word such that $p_{0} p_{1}=\mathrm{E}\left(p_{1}\right) u$. Necessarily $|u|=\left|p_{0}\right|$ and it follows from (13) that $u=(c \mathrm{E}(c))^{l_{0}} c$. Moreover, $u$ is a prefix of $E(\varphi(a))$. Since $u$ starts with $c$, it is not a prefix of $\mathrm{E}\left(p_{1}\right)$. Thus the penultimate letter $a$ in $w$ is necessarily 0 . Therefore $w=01 w^{\prime} 01=\Theta(0) w^{\prime} \Theta(0)$. We can easily see that $\varphi\left(w^{\prime}\right)$ is created from $\varphi(w)$ by removal of a prefix and suffix of length $|\varphi(01)|$. Thus $\varphi\left(w^{\prime}\right)$ is also an antipalindrome. The statement follows by induction on the length of $w$.

Finally, let us prove the statement (iii). Let $n=|v|$. By Lemma 33, we can find $w$ such that $\varphi(w)$ is an antipalindrome of length $|\varphi(w)|>r(n)(|\varphi(0)|+|\varphi(1)|)$, where $r(n)$ is the recurrence function of the uniformly recurrent word $\boldsymbol{u}$. Then $|w|>r(n)$ and $v$ is, therefore, a factor of $w$. Consequently, $v$ is a factor of $\Theta(u)$ for some $u \in\{0,1\}^{*}$, since $w=\Theta(u)$ by (ii). The uniqueness of the decoposition $v=x \Theta(z) y$ follows from the fact that a preimage under the Thue-Morse morphism is unique whenever 00 or 11 occur. The statement (iii) follows.

Lemma 41. Let $\varphi$ and $\boldsymbol{u}$ satisfy condition $(\star)$. Then there exist words $u^{(0)}$ and $u^{(1)}$ such that $\varphi(0)=\Theta\left(u^{(0)}\right)$ and $\varphi(1)=\Theta\left(u^{(1)}\right)$. 
Proof. By (i) of Lemma 40, $\varphi(0)$ contains either 00 or 11 as a factor. Thus using (iii) of the same lemma, the word $\varphi(0)$ can be uniquely written in the form $\varphi(0)=x \Theta(z) y$, where $x, y \in\{0,1, \varepsilon\}$. Since by Lemma 28 the language $\mathcal{L}(\boldsymbol{u})$ is closed under $\mathrm{E}$, we have $00,11 \in \mathcal{L}(\boldsymbol{u})$, and thus $\varphi(0) \varphi(0) \in \mathcal{L}(\boldsymbol{u})$. Again by (iii) of Lemma 40, $\varphi(0) \varphi(0)=$ $x^{\prime} \Theta\left(z^{\prime}\right) y^{\prime}$, where $x^{\prime}, y^{\prime} \in\{0,1, \varepsilon\}$ and the form $x^{\prime} \Theta\left(z^{\prime}\right) y^{\prime}$ is unique. Together, we have

$$
x^{\prime} \Theta\left(z^{\prime}\right) y^{\prime}=\varphi(0) \varphi(0)=x \Theta(z) y x \Theta(z) y .
$$

We split the discussion based on possible values of $x, y$ :

- If $x, y \neq \varepsilon$, then since $\varphi(0)$ is a palindrome, we have $x=y$, i.e., $x^{\prime} \Theta\left(z^{\prime}\right) y^{\prime}=$ $x \Theta(z) x x \Theta(z) x$. Since $x x$ cannot be the image under $\Theta$ of a letter, the situation is the following one (necessarily $x^{\prime}=y^{\prime}=\varepsilon$ )

\begin{tabular}{|c|c|c|c|c|c|c|c|c|}
\hline$x$ & \multicolumn{4}{|c|}{$\Theta(z)$} & $x$ & $x$ & \multicolumn{3}{|c|}{$\Theta(z)$} & $x$ \\
\hline \hline$\Theta\left(z_{1}^{\prime}\right)$ & $\cdots$ & $\Theta\left(z_{k-1}^{\prime}\right)$ & $\Theta\left(z_{k}^{\prime}\right)$ & $\Theta\left(z_{k+1}^{\prime}\right)$ & $\cdots$ & $\Theta\left(z_{2 k}^{\prime}\right)$ \\
\hline
\end{tabular}

and we have two different forms of $\varphi(0)=x \Theta(z) x=\Theta\left(z_{k+1}^{\prime} \cdots z_{2 k}^{\prime}\right)$, a contradiction.

- If $x \neq \varepsilon, y=\varepsilon$ then $x^{\prime} \Theta\left(z^{\prime}\right) y^{\prime}=x \Theta(z) x \Theta(z)$, the situation is either

\begin{tabular}{|c|c|c|c|c|c|}
\hline$x$ & $\Theta(z)$ & $x$ & \multicolumn{3}{|c|}{$\Theta(z)$} \\
\hline \hline$x^{\prime}$ & $\Theta\left(z_{1}^{\prime} \cdots z_{k}^{\prime}\right)$ & $\Theta\left(z_{k+1}^{\prime}\right)$ & $\cdots$ & $\Theta\left(z_{2 k}^{\prime}\right)$ & $y^{\prime}$ \\
\hline
\end{tabular}

or

\begin{tabular}{|c|c|c|c|c|c|c|}
\hline$x$ & \multicolumn{3}{|c|}{$\Theta(z)$} & \multicolumn{3}{|c|}{$\Theta(z)$} \\
\hline \hline$\Theta\left(z_{1}^{\prime}\right)$ & $\cdots$ & $\Theta\left(z_{k}^{\prime}\right)$ & $\Theta\left(z_{k+1}^{\prime}\right)$ & $\Theta\left(z_{k+2}^{\prime}\right)$ & $\cdots$ & $\Theta\left(z_{2 k+1}^{\prime}\right)$ \\
\hline
\end{tabular}

and again we have a contradiction with the uniqueness of the form of $\varphi(0)$ since either $\varphi(0)=x \Theta(z)=\Theta\left(z_{k+1}^{\prime} \cdots z_{2 k}^{\prime}\right) y^{\prime}$ or $\varphi(0)=x \Theta(z)=\Theta\left(z_{1}^{\prime} \cdots z_{k}^{\prime}\right) y^{\prime \prime}$, where $y^{\prime \prime}$ is the first letter of $\Theta\left(z_{k+1}^{\prime}\right)$.

- The case $x=\varepsilon, y \neq \varepsilon$ can be excluded analogously to the previous one.

Therefore $x=y=\varepsilon$ and $\varphi(0)=\Theta(z)$ for some $z \in\{0,1\}^{*}$. The same property of $\varphi(1)$ can be proved analogously.

Now we are in position to provide the proof of Theorem 36 .

Proof of Theorem 36. By (i) of Lemma 40 and Lemma 41 there is a palindrome $c$, words $u^{(0)}, u^{(1)}$, and $l_{0}, l_{1} \in \mathbb{N}$ such that

$$
\begin{aligned}
& \varphi(0)=\Theta\left(u^{(0)}\right)=(\mathrm{E}(c) c)^{l_{0}} \mathrm{E}(c), \\
& \varphi(1)=\Theta\left(u^{(1)}\right)=(c \mathrm{E}(c))^{l_{1}} c .
\end{aligned}
$$

Obviously, $|\varphi(0)|=2\left|u^{(0)}\right|=\left(2 l_{0}+1\right)|c|$. Thus $|c|$ is even and $\mathrm{E}(c)=\Theta\left(u_{1} u_{2} \cdots u_{n}\right)$, where $u_{1} u_{2} \cdots u_{n}$ is a prefix of $u^{(0)}$ such that $2 n=|\mathrm{E}(c)|$. Let us denote $\mathfrak{w}:=u_{1} u_{2} \cdots u_{n}$. As $c$ is a palindrome, $\mathrm{E}(c)$ is also a palindrome, and thus $\mathfrak{w}$ is indeed an antipalindrome. The statement of the theorem follows. 


\section{Summary and comments}

In this paper we have focused on morphisms with antipalindromic fixed points. Our aim was to define a suitable class of morphisms and study the analogy of the well known problem of Hof, Knill and Simon [10], known in the combinatorics on words as the HKS conjecture. We defined classes $\mathcal{A}_{1}, \mathcal{A}_{2}$ and demonstrated two main results for them. The question was previously studied by Labbé in [13. He proves that if a uniform morphism has an antipalindromic fixed point, then $\varphi$ or $\varphi \Theta$ is conjugated to a morphism in class $\mathcal{E}-\mathcal{P}$ defined by (2). He conjectures (Conjecture 5.5 in [13]) that in fact, always the latter is true. Taking into account the relation of classes $\mathcal{E}$-P and $\mathcal{A}_{1}$ in Remark 10 , our Theorem 34 proves this conjecture.

The main question about morphisms with antipalindormic fixed points remains open. We believe the following to be true.

Conjecture 42. Let $\varphi$ be a primitive binary morphism with an antipalindromic fixed point $\boldsymbol{u}$. Then $\varphi$ or $\varphi^{2}$ is conjugated to a morphism in class $\mathcal{A}_{1} \cup \mathcal{A}_{2}$.

Our Theorems 34 and 36 confirm the conjecture provided $\varphi$ is uniform or $\boldsymbol{u}$ is palindromic.

Let us look at the results of our paper yet from a different aspect. Theorem 34 can be seen as the analogy of the result of Tan [25] for uniform morphisms. We have shown that fixed points of morphisms in class $\mathcal{A}_{2} \backslash \mathcal{A}_{1}$ are not rigid, see Remarks 15 and 22 . Let us reformulate the statements of Theorems 34 and 36 with this aspect in mind.

Theorem 43. Let $\boldsymbol{u}$ be a fixed point of a primitive binary morphism which contains infinitely many both palindromes and antipalindromes.

- If $\boldsymbol{u}$ is rigid, then it is a fixed point of a uniform morphism of the form $\varphi(0)=w$, $\varphi(1)=E(w)$ for some $w \in\{0,1\}^{*}$.

- If $\boldsymbol{u}$ is not rigid, then it is a fixed point of a morphism in the form $\psi(0)=\Theta(w)$, $\psi(1)=\Theta\left((\mathrm{R}(w) w)^{l} \mathrm{R}(w)\right)$ where $w$ is an antipalindrome and $l \in \mathbb{N}$.

Our study motivates a number of questions for further research.

i) Infinite words containing arbitrarily long palindromes and antipalindromes can be constructed using the so-called palindromic and pseudopalindromic closure, introduced by de Luca and De Luca [5]. They showed the construction for the ThueMorse word. The details of the construction for complementary symmetric Rote words have been described in [3]. In [26], the authors study which of words generated by pseudopalindromic closure besides the Thue-Morse word $\boldsymbol{t}$ are fixed points of morphisms. They conjecture that only morphisms $\varphi:\{0,1\}^{*} \rightarrow\{0,1\}^{*}$ of the form

$$
\varphi(0)=0(110)^{k}, \quad \varphi(1)=1(001)^{k}, \quad k \in \mathbb{N}, k \geq 1,
$$

generate such fixed points. Let us mention that the above morphisms belong to both class $\mathcal{P}$ and $\mathcal{A}_{1}$. It would be interesting to clarify whether other morphisms in class $\mathcal{A}_{1}$ or $\mathcal{A}_{2}$ have fixed points arising by pseudopalindromic closure.

ii) Another way of constructing infinite words is given by the mapping $S:\{0,1\}^{\mathbb{N}} \rightarrow$ $\{0,1\}^{\mathbb{N}}$ defined by $S\left(u_{0} u_{1} u_{2} \cdots\right)=v_{0} v_{1} v_{2} \cdots$ where $v_{i}=u_{i}+u_{i+1}(\bmod 2)$ for $i \in \mathbb{N}$. If $S(\boldsymbol{u})$ contains infinitely many palindromes of odd length with central 
letter 1 , then the word $\boldsymbol{u}$ contains infinitely many antipalindromes. Mapping $S$ defines the relation between Sturmian words and complementary symmetric Rote words. In fact, $\boldsymbol{u}$ is a complementary symmetric Rote word if and only if $S(\boldsymbol{u})$ is a Sturmian word [22]. It would be interesting to determine for which $\boldsymbol{u}$ fixed point of a primitive morphism, the image $S(\boldsymbol{u})$ is also fixed by a primitive morphism and contains infinitely many palindromes with central letter 1 . An example of such a pair $\boldsymbol{u}$ and $S(\boldsymbol{u})$ is the Thue-Morse word $\boldsymbol{t}=01101001 \cdots$ and the fixed point $\boldsymbol{d}=1011101010111010 \cdots$ of the period-doubling morphism $D(0)=11, D(1)=10$. One can easily verify that $S(\boldsymbol{t})=\boldsymbol{d}$.

iii) Not all palindromic infinite words are rich in palindromes, in the sense of [6]. An example of an infinite word which is not rich is the Thue-Morse word $\boldsymbol{t}$. Nevertheless, $\boldsymbol{t}$ is generated by a morphism in class $\mathcal{P}$, namely $\Theta^{2}$. The question on which morphisms in class $\mathcal{P}$ have rich fixed point is not solved even for the binary case. Partial results about morphisms generating rich words is given in [8]. It is an interesting question to distinguish morphisms of classes $\mathcal{A}_{1} \cap \mathcal{P}, \mathcal{A}_{2} \cap \mathcal{P}$ such that their fixed points are $H$-rich, where $H$ is the group of morphisms and antimorphisms generated by $\mathrm{E}$ and $\mathrm{R}$, cf. [19].

iv) The antimorphism E defining antipalindromes in this article acts on the binary alphabet $\{0,1\}$. We may think of a generalization to multiletter alphabets $A$. Then one needs to consider a group $G$ generated by antimorphisms over the monoid $A^{*}$ and ask when an infnite word contains infinitely many $f$-palindromes for each antimorphims $f \in G$. Recall that an $f$-palindrome is a finite word $v \in A^{*}$ such that $f(v)=v$.

\section{Acknowledgments}

This work was supported by the project CZ.02.1.01/0.0/0.0/16_019/0000778 from European Regional Development Fund. We thank Štěpán Starosta for valuable comments.

\section{References}

[1] J.-P. Allouche, M. Baake, J. Cassaigne, D. Damanik, Palindrome complexity, Theoret. Comput. Sci. 292 (1) (2003) 9-31, doi:10.1016/S0304-3975(01) 00212-2.

[2] A. Blondin Massé, S. Brlek, S. Labbé, L. Vuillon, Palindromic complexity of codings of rotations, Theoret. Comput. Sci. 412 (46) (2011) 6455-6463, doi: $10.1016 /$ j.tcs. 2011.08 .007 .

[3] A. Blondin Massé, G. Paquin, H. Tremblay, L. Vuillon, On generalized pseudostandard words over binary alphabets, J. Integer Seq. 16 (2) (2013) Article 13.2.11, 28.

[4] S. Brlek, S. Hamel, M. Nivat, C. Reutenauer, On the palindromic complexity of infinite words, Internat. J. Found. Comput. Sci. 15 (2) (2004) 293-306, doi: 10.1142/S012905410400242X.

[5] A. de Luca, A. De Luca, Pseudopalindrome closure operators in free monoids, Theoret. Comput. Sci. 362 (1) (2006) 282-300, doi:10.1016/j.tcs.2006.07.009. 
[6] X. Droubay, J. Justin, G. Pirillo, Episturmian words and some constructions of de Luca and Rauzy, Theoret. Comput. Sci. 255 (1-2) (2001) 539-553, doi: 10.1016/S0304-3975(99)00320-5.

[7] N. P. Fogg, Substitutions in dynamics, arithmetics and combinatorics, vol. 1794 of Lecture Notes in Mathematics, Springer-Verlag, Berlin, doi:10.1007/b13861, 2002.

[8] A. Glen, J. Justin, S. Widmer, L. Q. Zamboni, Palindromic richness, European J. Combin. 30 (2) (2009) 510-531, doi:10.1016/j.ejc.2008.04.006.

[9] T. Harju, J. Vesti, L. Q. Zamboni, On a question of Hof, Knill and Simon on palindromic substitutive systems, Monatsh. Math. 179 (3) (2016) 379-388, doi: $10.1007 / \mathrm{s} 00605-015-0828-2$.

[10] A. Hof, O. Knill, B. Simon, Singular continuous spectrum for palindromic Schrödinger operators, Comm. Math. Phys. 174 (1) (1995) 149-159, URL: http://projecteuclid.org/euclid.cmp/1104275098.

[11] K. Klouda, Bispecial factors in circular non-pushy D0L languages, Theoret. Comput. Sci. 445 (2012) 63-74, doi:10.1016/j.tcs.2012.05.007.

[12] D. Krieger, On stabilizers of infinite words, Theoret. Comput. Sci. 400 (1-3) (2008) 169-181, doi:10.1016/j.tcs.2008.03.017.

[13] S. Labbé, Propriétés combinatoires des f-palindromes, Master's thesis, Université du Québec à Montréal, 2008.

[14] S. Labbé, E. Pelantová, Palindromic sequences generated from marked morphisms, European J. Combin. 51 (2016) 200-214, doi:10.1016/j.ejc.2015.05.006.

[15] M. Lothaire, Combinatorics on words, Cambridge Mathematical Library, Cambridge University Press, Cambridge, doi:10.1017/CB09780511566097, 1997.

[16] Z. Masáková, E. Pelantová, Š. Starosta, Exchange of three intervals: substitutions and palindromicity, European J. Combin. 62 (2017) 217-231, doi: 10.1016/j.ejc.2017.01.003.

[17] K. Medková, E. Pelantová, L. Vuillon, Derivated sequences of complementary symmetric Rote sequences, submitted to RAIRO - Theor. Inf. Appl., 2018, URL: https://arxiv.org/abs/1812.03748.

[18] J. Pansiot, The morse sequence and iterated morphisms, Inf. Process. Lett. 12 (2) (1981) 68-70, doi:10.1016/0020-0190(81)90004-1.

[19] E. Pelantová, Š. Starosta, Languages invariant under more symmetries: overlapping factors versus palindromic richness, Discrete Math. 313 (21) (2013) 2432-2445, doi: $10.1016 / j$.disc. 2013.07 .002 .

[20] E. Pelantová, Š. Starosta, Constructions of words rich in palindromes and pseudopalindromes, Discrete Mathematics \& Theoretical Computer Science 18 (3) (2016) \#16, URL: https://dmtcs.episciences.org/2202. 
[21] M. Queffélec, Substitution dynamical systems - Spectral analysis, vol. 1294 of Lecture Notes in Mathematics, Springer-Verlag, Berlin, second edn., doi: 10.1007/978-3-642-11212-6, 2010.

[22] G. Rote, Sequences with subword complexity 2n, J. Number Theory 46 (2) (1994) 196-213, doi:10.1006/jnth.1994.1012.

[23] P. Séébold, On the conjugation of standard morphisms, Theoret. Comput. Sci. 195 (1) (1998) 91-109, doi:10.1016/S0304-3975(97)00159-X.

[24] P. Séébold, About some overlap-free morphisms on a $n$-letter alphabet, J. Autom. Lang. Comb. 7 (4) (2002) 579-597.

[25] B. Tan, Mirror substitutions and palindromic sequences, Theoret. Comput. Sci. 389 (1-2) (2007) 118-124, doi:10.1016/j.tcs.2007.08.003.

[26] T. Velká, L’. Dvořáková, Fixed points of morphisms among binary generalized pseudostandard words, Integers 18 (2018) Paper No. A21, 9. 